\title{
Desarrollo de un nuevo semiremolque extensible, para ser usado como plató móvil, construido en acero de alta resistencia.
}

\author{
David Valladares ${ }^{[1]}$, Luis Castejón ${ }^{[1]}$, Juan Marquesán ${ }^{[1]}$, Juan Carlos Salas ${ }^{[2]}$, Hugo \\ Malón ${ }^{[1]}$ \\ [1] Departamento de Ingeniería Mecánica, Universidad de Zaragoza \\ [2] Alegría Activity S.L.
}

\section{RESUMEN}

En el presente artículo se expone el proceso llevado a cabo para desarrollar un nuevo vehículo extensible para servicios públicos. Consiste en un semirremolque que dispone de un circuito de elementos hidráulicos que le permiten desplegar varias extensiones y un planta superior, una vez que se encuentra estático y fijado sobre unas patas de apoyo. Sin embargo, durante los trayectos que realice el semirremolque, el piso superior y todos los ventanales y extensiones del vehículo deben estar completamente replegados y recogidos, de tal forma que las dimensiones exteriores del vehículo se adecuen a las exigencias reglamentarias para su libre circulación por carretera. Por todo ello se hace necesario garantizar un funcionamiento adecuado y seguro del vehículo tanto durante las maniobras hidráulicas de extensión y recogida, como una vez desplegado y listo para ser utilizado, por ejemplo, como centro operativo para la cobertura de eventos por parte de televisiones $u$ otros medios de comunicación.

En primer lugar se identificaron como zonas estructurales más críticas a esfuerzos las correspondientes al accionamiento de los techos abatibles del primer y del segundo nivel del semirremolque. A continuación, se crearon modelos numéricos de malla de estas zonas y se analizaron por el método de los elementos finitos. A partir de los resultados obtenidos se procedió a optimizar la estructura para mejorar su comportamiento mecánico e incrementar el coeficiente de seguridad frente a posibles deformaciones plásticas, al mismo tiempo que se redujo en lo posible el peso del vehículo utilizando aceros de alta resistencia en lugar de aceros tradicionales.

Se realizó además un análisis numérico de la estructura completa del vehículo para comprobar el comportamiento ante solicitaciones exteriores como el viento o una sobrecarga por exceso de número de personas en el interior de los habitáculos del vehículo. Finalmente se llevaron a cabo ensayos extensiométricos sobre la estructura del vehículo fabricado. Se obtuvo una adecuada correlación numérico-experimental que permitió validar los resultados numéricos obtenidos. 


\section{OBJETIVOS PLANTEADOS}

Por medio del presente artículo se desarrollado una metodología de cálculo numérico, optimización y ensayo de semirremolques especiales con partes extensibles, aplicables a diferentes servicios públicos, que sea capaz de determinar las zonas estructuralmente críticas del vehículo y las maniobras más desfavorables. Para ello, se aplicó el procedimiento planteado al cálculo y optimización de un semirremolque extensible que será utilizado como centro de control y plató televisivo para eventos deportivos. A continuación se construyó un prototipo y se realizaron a cabo ensayos de validación del mismo. Se comprobó el comportamiento global del vehículo ante casos de carga correspondientes a los movimientos de extensión de las partes extraíbles y otros casos actuantes como viento o reposo. El vehículo objeto del proyecto ha sido diseñado y construido por la empresa ALEGRÍA ACTIVITY S.L.

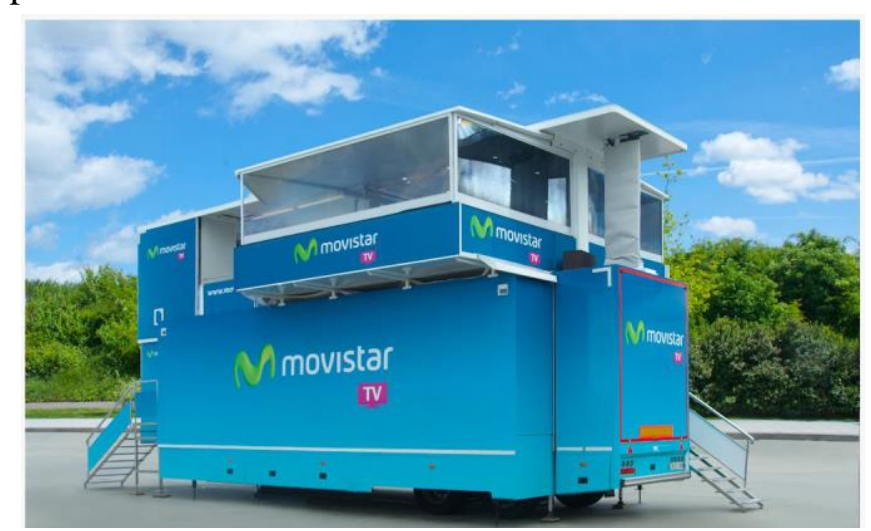

Figura 1. Vista de semirremolque desplegado

\section{METODOLOGÍA DESARROLLADA}

El proceso seguido en el desarrollo de esta metodología se detalla a continuación.

A partir de la experiencia de la empresa en un diseño previo con características similares se identificó como zona más crítica a esfuerzos la correspondiente a los techos abatibles tanto del primer como del segundo nivel del semirremolque. Estos, en el momento en que quedaban completamente elevados, sufrían fallos puntuales y deformaciones excesivas que llevaron a la empresa a plantearse un estudio de esa parte del vehículo. Dada la similitud de diseño observada entre la estructura de elevación de los ventanales inferiores (techo de la primera planta y suelo de la segunda planta) y la estructura de elevación de los ventanales superiores (techo de la segunda planta) se decidió estudiar únicamente la estructura correspondiente al techo superior y los ventanales superiores. Los resultados obtenidos son por tanto extensibles al diseño de los ventanales inferiores.

En definitiva se estudiaron: 
- Estructura de ventanal superior: Las zonas de conexión de los cuatro cilindros hidráulicos con los ventanales de techo para realizar la maniobra de elevación y bajada de los mismos.

- Estructura de techo superior: Las zonas de apoyo de los cuatro cilindros hidráulicos sobre los perfiles del techo superior para realizar elevación de los ventanales de techo.

Para su estudio, el grupo VEHIVIAL empleó modernas técnicas de análisis numérico basadas en el método de elementos finitos. Para ello se crearon representaciones virtuales de la zona en cuestión utilizando software de diseño asistido por ordenador y posteriormente modelos de malla capaces de discretizar la estructura en un conjunto de elementos (planos bidimensionales tipo "Shell" o barras unidimensionales tipo "Beam") conectados por nodos a los que se asignan los propiedades correspondientes de material y sección resistente. A partir de los planos en AutoCAD aportados por la empresa a VEHIVIAL fue posible realizar los modelos virtuales tridimensionales de elementos finitos correspondientes, de acuerdo a la geometría de las zonas críticas del semirremolque a estudiar que se han indicado.

A partir de los resultados obtenidos se propusieron y plantearon modificaciones para la mejora del comportamiento y de la seguridad del conjunto estructural.

Se realizó también un análisis numérico de la estructura completa del vehículo para comprobar el comportamiento ante solicitaciones exteriores como el viento o una sobrecarga por exceso de número de personas en el interior de los habitáculos del vehículo. Se modelizó numéricamente un modelo global del semirremolque de gran complejidad utilizando elementos barra tipo "beam". De esta forma fue posible estudiar casos de carga desfavorables, de cara a la seguridad estructural, que a nivel de submodelos de zonas concretas no sería posible simular.

Finalmente para garantizar la fiabilidad de los resultados numéricos obtenidos se llevaron a cabo ensayos extensométicos sobre la estructura del vehículo, una vez fabricada, y se compararon los resultados experimentales con los numéricos. Se obtuvo una adecuada correlación numérico-experimental en los puntos de medida de deformación que fueron seleccionados. 


\section{ANÁLISIS POR EL M.E.F. DE LA ESTRUCTURA DE TECHO Y VENTANAL SUPERIORES}

Para llevar a cabo este análisis se han seguido los siguientes pasos:

\subsection{Cálculo del ventanal de techo elevado y sujeto por los cilindros recogidos.}

Dado que el ventanal es idéntico a cada lado del vehículo y dada la simetría del ventanal, se simplificó el modelo a medio ventanal sujeto por dos cilindros hidráulicos. Se utilizaron elementos planos tridimensionales "shell" de tres y cuatro nodos en el modelo y a partir de este cálculo fue posible determinar el estado tensional del ventanal de techo en su posición más elevada y también más desfavorable. Se emplearon masas puntuales adicionales para simular el peso del acristalamiento. De este modo se obtuvieron tanto las fuerzas necesarias en cada cilindro para sujetar el ventanal en esta posición como las reacciones en las bisagras sobre las que gira el ventanal. Estas fuerzas y reacciones se aplicaron al siguiente paso de cálculo del techo indicado a continuación.

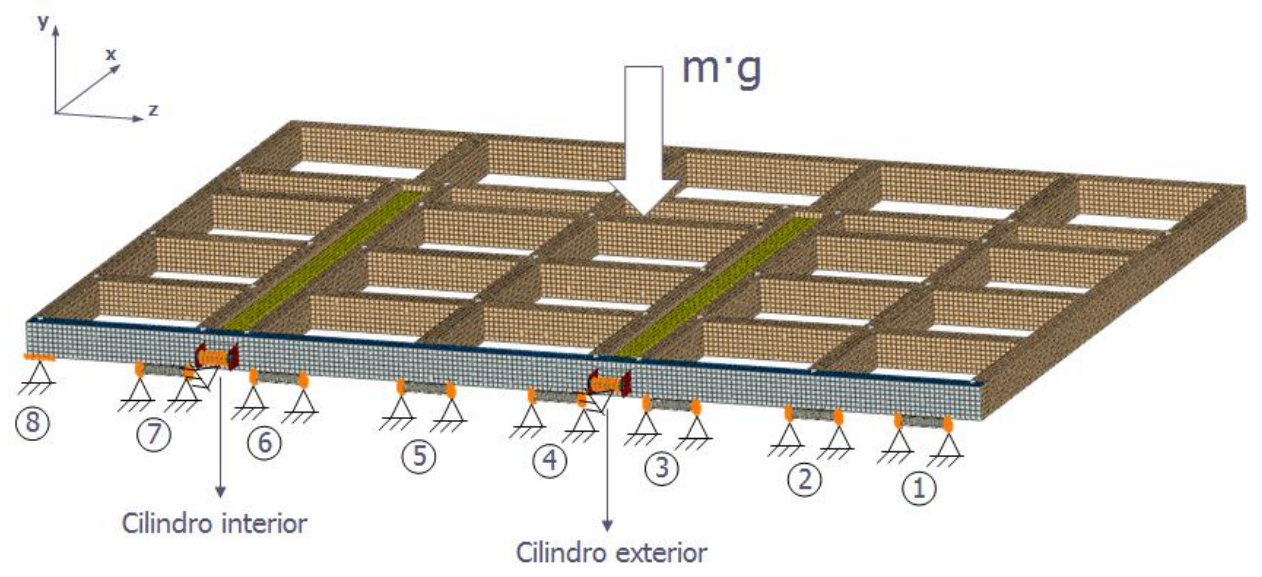

Figura 2. Modelo numérico de la mitad del ventanal superior

\subsection{Cálculo del acoplamiento del cilindro a la estructura central del techo para el caso de carga de ventanal de techo elevado.}

Dada la similitud de cada acoplamiento de cada cilindro hidráulico al techo se realizó un modelo reducido o submodelo de la zona de acoplamiento, conteniendo un tramo del techo correspondiente a un cilindro hidráulico y dos bisagras de apoyo del ventanal. Se utilizaron elementos planos tridimensionales "shell" de tres y cuatro nodos en el modelo al cual fue posible aplicarle la fuerza de cilindro más elevada de entre las dos obtenidas en el modelo de ventanal (la del cilindro más exterior del ventanal alcanzó el valor superior de $45687 \mathrm{~N}$ ) así como las fuerzas correspondientes al apoyo del ventanal sobre las dos bisagras del techo presentes en el submodelo (respectivamente 20968 N y 21600 N en dirección 
transversal hacia dentro y $495 \mathrm{~N}$ y $1095 \mathrm{~N}$ en dirección vertical hacia abajo).

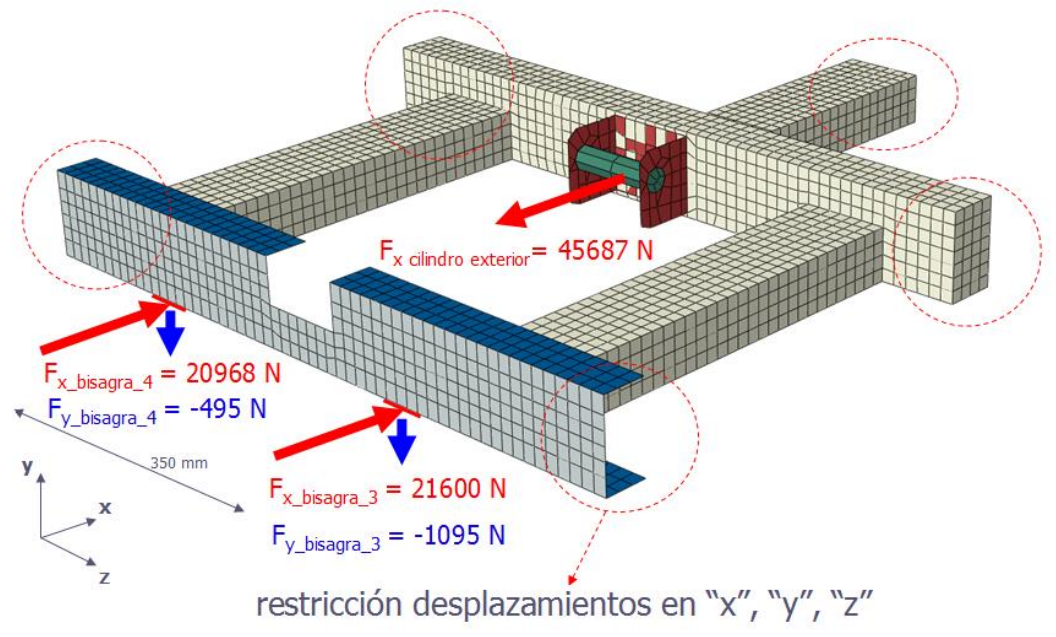

Figura 3. Modelo numérico de tramo techo superior

\subsection{Cálculo a rigidez de la zona central de la estructura del techo superior para caso de ventanales de techo elevados.}

Los puntos 3.1 y 3.2 anteriores corresponden al estudio a resistencia de la estructura de techo. Para llevar a cabo un estudio a rigidez o del nivel de deformación de todo el tramo estructural del techo se realizó un tercer modelo en elementos barra unidimensionales tipo "beam" de la zona central de la estructura del techo superior sobre la que se acoplan los ocho cilindros hidráulicos encargados de la maniobra de los ventanales (cuatro en cada ventanal). Se trata de un modelo con menor nivel de detalle que los anteriores que sin embargo permitió analizar el comportamiento a rigidez del conjunto de la estructura central incorporando todas las fuerzas de los cilindros y de los apoyos de las bisagras de los ventanales superiores.

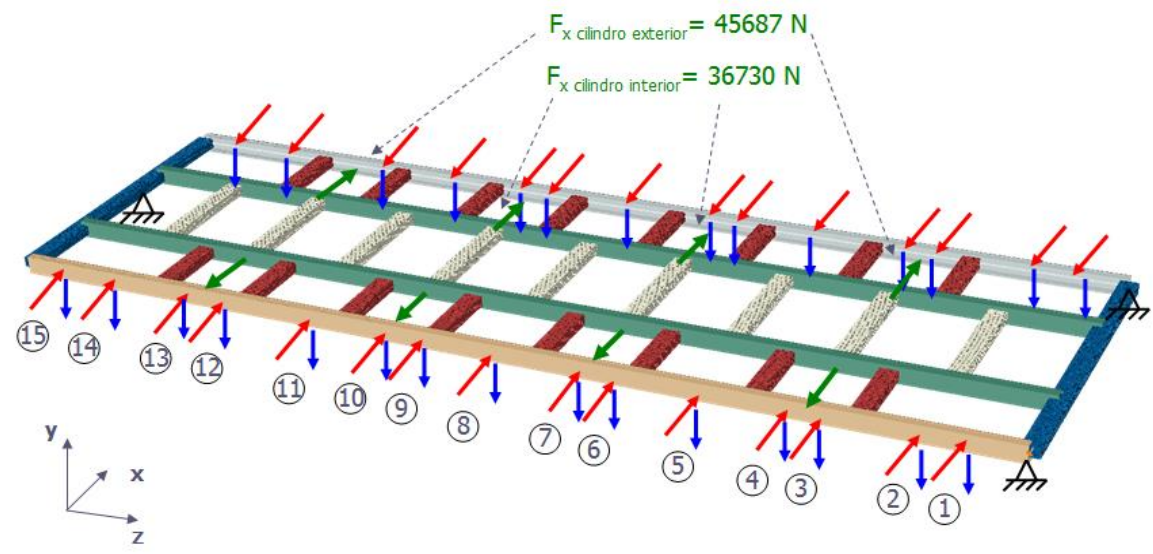

Figura 4. Modelo numérico del techo superior

\section{OPTIMIZACIÓN ESTRUCTURAL}

Tras el análisis de los resultados se procedió a optimizar la estructura para mejorar su comportamiento mecánico e incrementar el coeficiente de seguridad frente a posibles 
deformaciones plásticas, al mismo tiempo que se reduce en lo posible el peso del vehículo. La estrategia seguida en este caso consistió en:

- Empleo de aceros especiales de alto límite elástico. En concreto se utilizó acero DOMEX 700 MC, en lugar del acero estandar S235JR planteado en el diseño inicial.

- Empleo de perfiles de tubo hueco de sección rectangular con menores dimensiones exteriores a los propuestos inicialmente: Se sustituyeron perfiles 60x100x4 de acero S235JR por perfiles 50x100x5 mm de acero DOMEX $700 \mathrm{MC}$ en las zonas más conflictivas con tensiones equivalentes más elevadas y por perfiles $60 \times 100 \times 2$ de acero S235JR en las zonas menos solicitadas a tensión.

De esta forma se obtuvieron los siguientes resultados:

- Se han obtenido coeficientes de seguridad con valor 1.97 en los nuevos perfiles en Domex 700MC, de rango muy similares al valor de 2.1 obtenido en el modelo anterior diseñado integramente en S235JR.

- Se ha reducido el peso del ventanal completo en $36.6 \mathrm{~kg}$, esto propiciará que la fuerza que soporten los cuatro cilindros de apoyo sea menor y por tanto se reducirán los esfuerzos en los perfiles del techo.

- El desplazamiento vertical máximo obtenido es menor, es decir, se ha aumentado la rigidez del modelo, pasando de $6.6 \mathrm{~mm}$ a $1.9 \mathrm{~mm}$ en el extremo exterior.

\section{ANÁLISIS GLOBAL DE LA ESTRUCTURA}

Para garantizar un comportamiento seguro de todo el conjunto estructural ante posibles solicitaciones exteriores que pueden tener lugar, se estudió también el comportamiento global de la estructura desplegada completamente y apoyada sobre sus patas ante las siguientes solicitaciones:

- Carga de viento sobre la superficie de incidencia más desfavorable: se ha aplicado una presión de $80 \mathrm{~kg} / \mathrm{m}^{2}$ en todo el flanco lateral de $72.58 \mathrm{~m}^{2}$.

- Carga de sobrepeso por la presencia de un excesivo número personas en las dos plantas: se ha considerado una carga de $200 \mathrm{~kg} / \mathrm{m}^{2}$ en toda la planta inferior y superior $\left(70 \mathrm{~m}^{2}\right.$ en total).

Se realizó para ello un modelo completo del vehículo en elementos barra tipo "beam" y se analizaron los casos de carga indicados. A continuación, se muestran en las figuras 5 y 6 los desplazamientos obtenidos en ambos casos. 


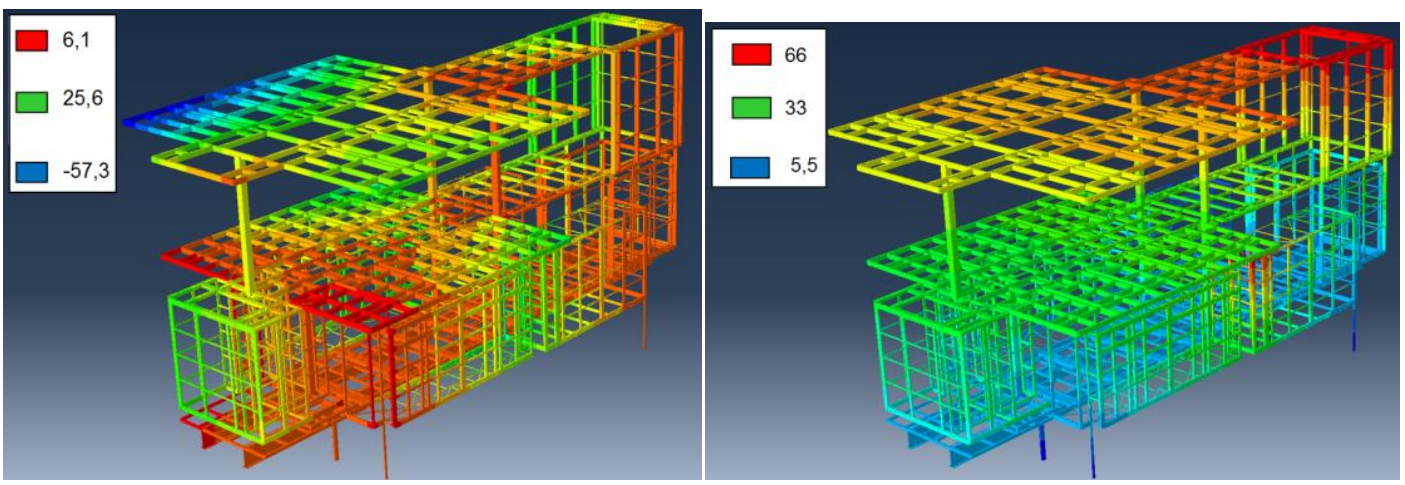

Figura 5. Izda. Desplazamiento vertical para carga de viento lateral. Drcha. Desplazamiento transversal para carga de viento lateral ( $\mathrm{mm}$ )
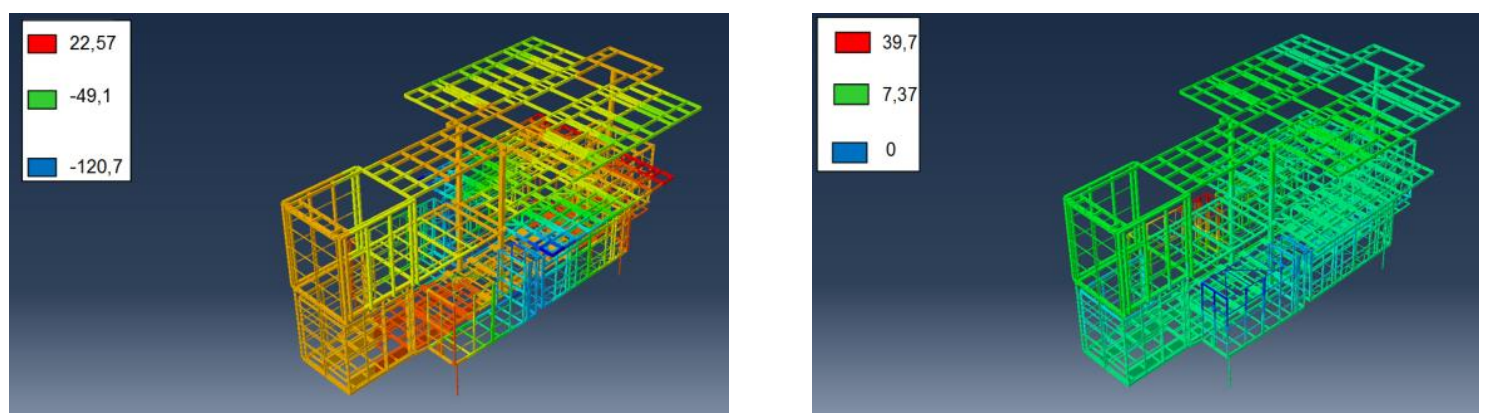

Figura 6. Izda. Desplazamiento vertical para caso de sobrecarga de personas. Drcha. Desplazamiento transversal para caso de sobrecarga de personas ( $\mathrm{mm}$ )

\section{REALIZACIÓN DE ENSAYOS DE EXTENSOMETRÍA}

Finalmente se realizaron ensayos entensométricos para medir el nivel de deformación y determinar el estado tensional alcanzado en la maniobra de elevación del ventanal superior en unas zonas concretas y comparar el resultado con el modelo de elementos finitos calculados. Se utilizaron dos galgas extensiométicas para la medida para las cuales se obtuvo una correcta correlación numérico-experimental en los puntos de medida seleccionados, lo cual garantizó un nivel de aproximación y fiabilidad adecuado en la metodología desarrollada.
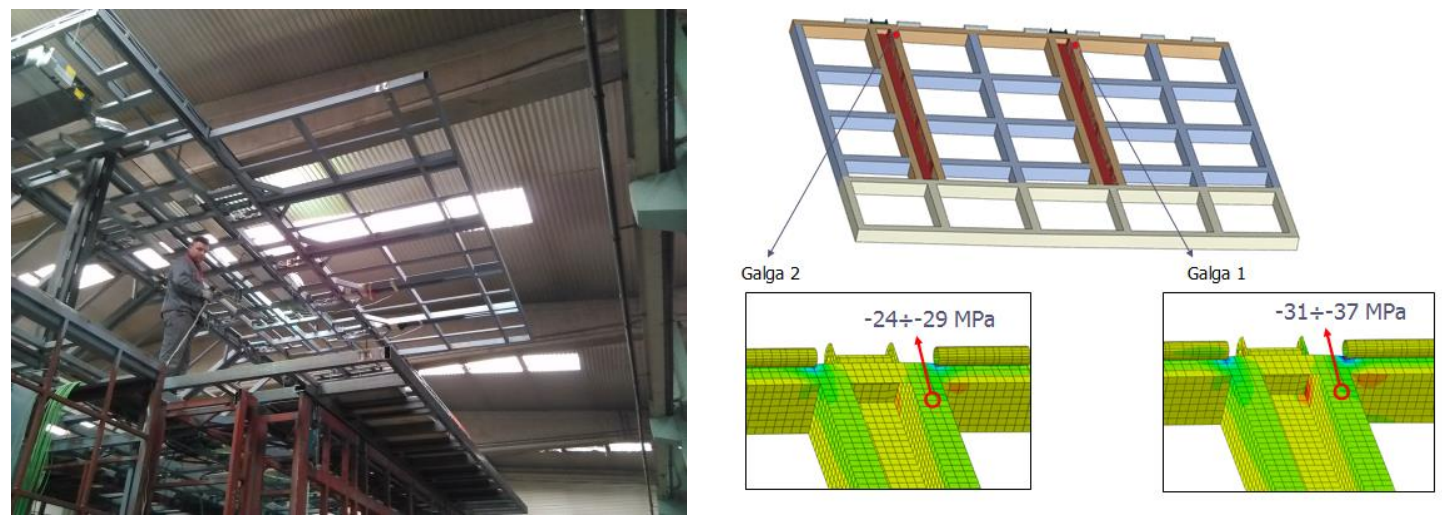

Figura 7. Ensayo de elevación del ventanal superior y simulación numérica del mismo 
$\mu \varepsilon$ (microdeformación)

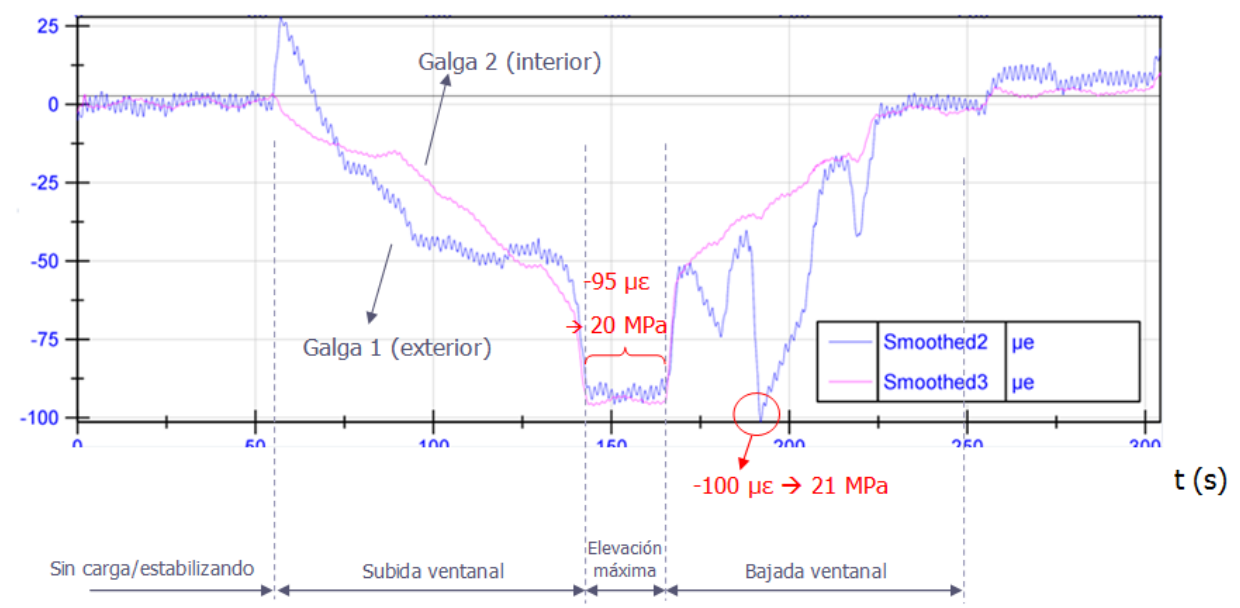

Figura 8. Resultado del ensayo de extensometría en galgas 1 y 2.

\section{AGRADECIMIENTOS}

Este proyecto ha sido desarrollado para la empresa Alegría Activity S.L. en el marco del proyecto "Desarrollo de una metodología de cálculo y optimización de unidades móviles para servicios públicos", financiado a través de la convocatoria DPZ, de ayudas para el desarrollo de proyectos de $\mathrm{I}+\mathrm{D}+\mathrm{i} 2014$.

\section{REFERENCIAS}

CARrerA, M., CASTEJÓN, L., GIL, E., OLMOS, J.MA., LARRODÉ E., NUEZ C., MARTÍN, C., FABRA, C. (2004). Development of an innovative concept of light semitrailer by means of FEM and testing, Achieving Lightweight Vehicles, SP-1846, SAE Internacional, March, ISBN 0-7680-1382-8.

CARRERA, M. Desarrollo de conceptos innovadores de semirremolques mediante la aplicación de técnicas numéricas y experimentales. Diseño de una bancada de ensayos de fatiga para su simulación frente a maniobras críticas. Tesis doctoral. Universidad de Zaragoza. 2006.

LARRODÉ, E., MIRAVETE A., AND CASTEJÓN, L. (1998), Design and Optimisation of a Lightweight Frigorific Semitrailer. Heavy Vehicle Systems.-International Journal of Vehicle Design (U.K.), 1998 Vol. 5, № 1, pp. 45-67. ISSN. 1351-7848.

MALÓN, H. Desarrollo de un método innovador de análisis de comportamiento frente a cargas de fatiga de uniones soldadas y componentes estructurales de semirremolque. Tesis doctoral. Universidad de Zaragoza. 2010.

ZIENKIEWICS, O.C., TAYLOR, R.L. (1994). El método de los elementos finitos. Volumen 1 y 2. Ed. CIMNE, Barcelona, $4^{\mathrm{a}}$ edition. 